\title{
The near-infrared reflected spectrum of source I in Orion-KL ${ }^{\star}$
}

\author{
L. Testi ${ }^{1,2}$, J. C. Tan ${ }^{3}$, and F. Palla ${ }^{2}$ \\ 1 ESO, Karl Schwarzschild str. 2, 85748 Garching, Germany \\ e-mail: 1testi@eso.org \\ 2 INAF-Osservatorio Astrofisico di Arcetri, Largo E. Fermi 5, 50125 Firenze, Italy \\ 3 Departments of Astronomy \& Physics, University of Florida, Gainesville, FL 32611, USA
}

Received 24 March 2010 / Accepted 31 May 2010

\section{ABSTRACT}

\begin{abstract}
Context. Source I in the Orion-KL nebula is believed to be the nearest example of a massive star still in the main accretion phase. It is thus one of the best cases for studying the properties of massive protostars to constrain high-mass star formation theories. Nearinfrared radiation from source I escapes through the cavity opened by the OMC1 outflow and is scattered by dust towards our line of sight.

Aims. The reflected spectrum offers a unique possibility of observing the emission from the innermost regions of the system and probing the nature of source I and its immediate surroundings.

Methods. We obtained moderately high spectral-resolution $(\lambda / \Delta \lambda \sim 9000)$ observations of the near infrared diffuse emission in several locations around source I/Orion-KL. We observed a widespread rich absorption line spectrum that we compare with cool stellar photospheres and protostellar accretion disk models.

Results. The spectrum is broadly similar to strongly veiled, cool, low-gravity stellar photospheres in the range $T_{\text {eff }} \sim 3500-4500 \mathrm{~K}$, luminosity class I-III. An exact match explaining all features has not been found, and a plausible explanation is that a range of different temperatures contribute to the observed absorption spectrum. The 1D velocity dispersions implied by the absorption spectra, $\sigma \sim 30 \mathrm{~km} \mathrm{~s}^{-1}$, can be explained by the emission from a disk around a massive, $m_{*} \sim 10 M_{\odot}$, protostar that is accreting at a high rate, $\dot{m}_{*} \sim 3 \times 10^{-3} M_{\odot} \mathrm{yr}^{-1}$.

Conclusions. Our observations suggest that the near-infrared reflection spectrum observed in the Orion-KL region is produced close to source I and scattered to our line of sight in the OMC1 outflow cavity. The spectrum allows us to exclude source I being a very large, massive protostar rotating at breakup speed. We suggest that the absorption spectrum is produced in a disk surrounding a $\sim 10 M_{\odot}$ protostar, accreting from its disk at a high rate of a few $\times 10^{-3} M_{\odot} / \mathrm{yr}$ ).
\end{abstract}

Key words. stars: formation - accretion, accretion disks - stars: protostars - stars: massive

\section{Introduction}

Our understanding of massive star formation is still very limited compared to that of solar-mass stars, for which a generally accepted theoretical framework exists (Shu et al. 1987). The roles of dynamical interactions and coalescence versus a more standard core and disk accretion framework are still being debated (Beuther et al. 2007; Zinnecker \& Yorke 2007). Progress has been limited in part by the greater observational difficulties of studying massive protostars that are typically more distant, embedded, crowded, and quickly evolving than their lower mass cousins. Orion is the closest star-forming region where young, massive $\left(M \geq 8 M_{\odot}\right)$ stars are present, and as such is an ideal test case to study observationally and to understand theoretically.

In particular, the Kleinman-Low (KL) nebula and the Becklin-Neugebauer $(\mathrm{BN})$ object have received a lot of attention since their discovery as infrared sources (Becklin \& Neugebauer 1967; Kleinmann \& Low 1967). Rieke et al. (1973) resolved several point sources in the KL nebula, and it was soon recognized that at least some of these objects were young massive stars. Subsequent higher angular resolution, infrared observations showed that only a limited number of these sources were self-luminous objects. Infrared polarimetric observations of the

* Based on observations collected at the European Southern Observatory, Chile. Program 076.C-0660. region have shown that one source is responsible for the illumination of most of the nebula, with the only exception being a small area around BN (Minchin et al. 1991). Radio continuum observations have identified three sources that are likely young (possibly still forming) massive stars in the region: source $\mathrm{n}$ (also associated with an infrared source), BN, and source I, located near the Orion hot molecular core and near the infrared source IRc2 (Menten \& Reid 1995). The mid-IR spectrum of source $\mathrm{n}$ and its relatively low extinction imply that it is not particularly luminous (Gezari et al. 1998). Similarly, the polarization data of Minchin et al. (1991) and Werner et al. (1983) indicate $\mathrm{BN}$ is not responsible for most of the luminosity of the $\mathrm{KL}$ nebula. $\mathrm{BN}$ is known to be a runaway star (Plambeck et al. $1995)$, moving through the ONC at about $30 \mathrm{~km} \mathrm{~s}^{-1}$. In one scenario, it was ejected from near the Trapezium region by dynamical interaction with the $\theta^{1} C$ binary (Tan 2008, 2004), and thus would have made a close passage to source I, perhaps enhancing its accretion rate by tidal interaction. In an alternative scenario, BN was ejected by dynamical interaction with source I itself (Bally \& Zinnecker 2005; Gómez et al. 2008). Source n may also have been involved in this interaction. This scenario requires source I to be composed of a massive, compact binary, with total mass more than twice the mass of BN, i.e. $\gtrsim 20 M_{\odot}$.

In either case, source $I$ is thus expected to be the main illuminating source of the infrared nebula. It is associated with 


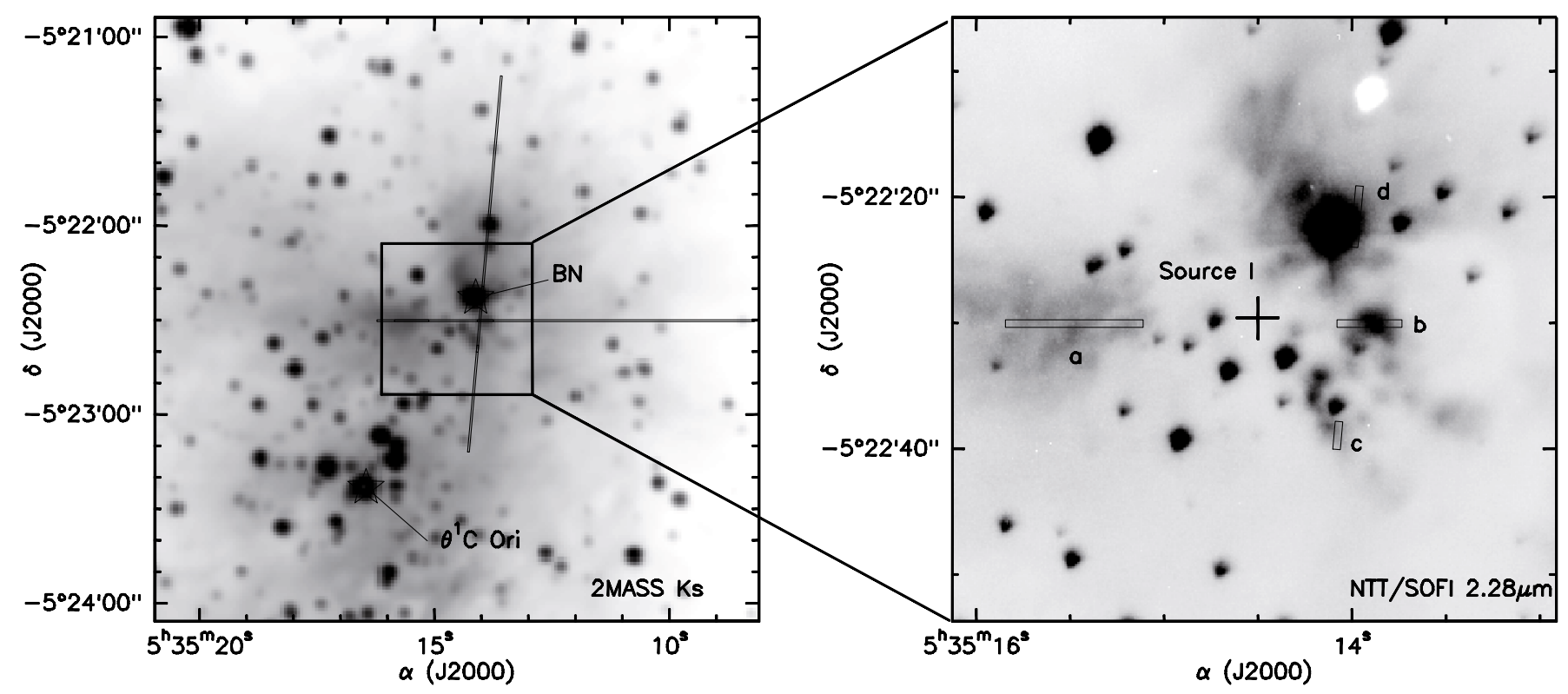

Fig. 1. Left panel: 2 MASS $K$ s-band image of the Orion Trapezium and the Kleinmann-Low nebula region, $\theta^{1} \mathrm{C}$ and the Becklin-Neugebauer object are marked for reference. The positions of the two on-source slits are shown. Right panel: the region around Orion-KL source I (marked with a cross and labeled) at $2.28 \mu \mathrm{m}$ is reproduced from an ESO-Archive SOFI-NTT observation (originally acquired for the ESO program 64.I-0493). The small slitlets marked from a to d show the regions we used to extract the spectra shown in Fig. 2.

an outflow detected in $\mathrm{SiO}$ and compact radio emission believed to originate in either a small ionized disk (Goddi et al. 2009; Plambeck et al. 1990, 2009) or in the ionized base of an outflow cavity (Tan \& McKee 2003). The source is deeply embedded and is not revealed at near infrared and shorter wavelengths. Nevertheless, the surrounding reflection nebula offers a unique opportunity to observe the reflected emission from the heart of the system. Morino et al. (1998) were the first to obtain a low spectral resolution, near infrared $(2.05-2.35 \mu \mathrm{m})$ spectrum of the reflected light from source I. They revealed that the reflected spectrum shows the absorption lines typical of a cool photosphere, including metal lines and the $\mathrm{CO}(2-0)$ overtone absorption starting at $2.29 \mu \mathrm{m}$. The inferred equivalent photospheric temperature $\left(T_{\star} \sim 4500 \mathrm{~K}\right)$ and derived luminosity $\left(L_{\star} \sim 10^{5} L_{\odot}\right)$ could be consistent with either a very large, cool, massive protostar or the "photosphere" of a very active accretion disk. Nakano et al. (2000) discus the first possibility, using one-zone protostellar models and concluding that the required size of $\gtrsim 300 R_{\odot}$ could not be achieved by reasonable accretion rates, even up to $10^{-2} M_{\odot} \mathrm{yr}^{-1}$. They suggest that the large size could be attained if the protostar was rotating at near the breakup speed, which would then imply very broadened line profiles. Subsequent work involving multi-zone protostellar models by Yorke \& Bodenheimer (2008) and Hosokawa \& Omukai (2009) has shown that the sizes can be about a factor of several larger than those predicted by the one-zone models during the (relatively brief) luminosity wave expansion phase (Stahler et al. 1986) of the evolution. Hosokawa \& Omukai (2009) conclude that the low temperature implied by the infrared observations of the reflected spectrum from source I in Orion-KL could be achieved if the accretion rate was $\gtrsim 4 \times 10^{-3} M_{\odot} \mathrm{yr}^{-1}$, averaged over the entire formation time of the protostar.

However, such average accretion rates are at least an order of magnitude greater than those expected if massive stars form from massive cores in near equilibrium with the pressures in the Orion Nebula region (McKee \& Tan 2003). Since a massive protostar will be surrounded by a relatively cool (compared to the protostar), optically thick accretion disk, we think that this is a more likely source of the observed near IR reflection nebula. The various models can be tested by resolving the profiles of the absorption lines. With this in mind we carried out intermediate-resolution $(R \sim 8900)$, near-infrared observations of the reflected spectrum from source I in Orion-KL.

\section{Observations and data reduction}

Near-infrared medium resolution spectra of the scattered light around the Orion-KL region were obtained using the ISAAC near-infrared camera and spectrograph at the ESO-VLT UT1 telescope. The observations were carried out in service mode on several occasions during October-November 2005. We used the 0.3 arcsec slit, and the medium-resolution grism that offered a resolution of $\sim 8900$ across the wavelength range $2.23-2.33 \mu \mathrm{m}$. The observing sequence was composed of sets of on-source and nearby (10 arcmin away) blank sky spectra arranged as standard ABBA cycles, to allow for efficient and accurate sky subtraction. For each of the two slit positions (see below and Fig. 1), we integrated a total of $40 \mathrm{~min}$ on source. Standard calibrations (flats and lamps) and telluric standards spectra were obtained for each observation as part of the ISAAC calibration plan. Wavelength calibration was performed using the lamp observations and refined using the bright $\mathrm{OH}$ sky lines.

The spectra were reduced using standard procedures in IRAF. After checking that the data sets obtained in different nights were not significantly different, we combined all the exposures to obtain a final spectrum for each of the two slit positions shown in the left panel of Fig. 1.

\section{Results}

Spectra were extracted for four regions corresponding to the brightest parts of the reflection nebulosity within the observed slits (see Fig. 1 for a detailed chart). Region "a" corresponds to the region observed by Morino et al. (1998); regions "b" and "c" correspond to two bright nebulosity patches to the west and to 


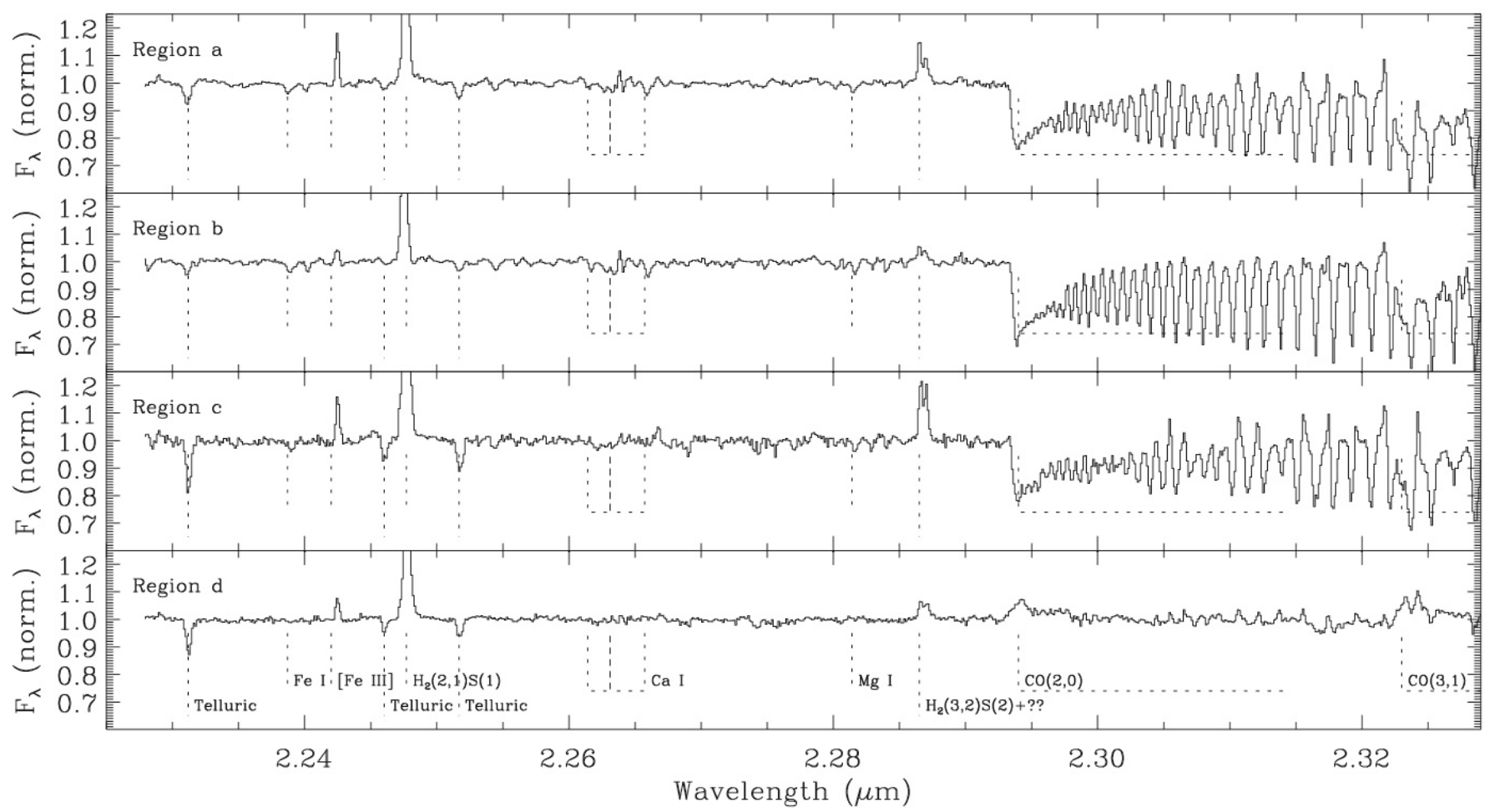

Fig. 2. From top to bottom: final spectra of the four regions a to d shown in Fig. 1. Each spectrum has been normalized to the continuum level. Some of the most prominent emission or absorption features are labeled. The features labeled "Telluric" are negative residuals from the subtraction of the $\mathrm{OH}$ airglow spectrum. The spectra also show the prominent emission features of $\mathrm{H}_{2}$ and [FeIII] which originate from diffuse gas in the region and (most likely) not associated with the reflection nebulosity.

the south of the source I position, respectively; and region " $\mathrm{d}$ " is very close to the BN object.

All spectra show emission lines of $\mathrm{H}_{2}(2,1) \mathrm{S}(1), \mathrm{H}_{2}(3,2) \mathrm{S}(2)$ (blended with an unidentified infrared emission feature), and [FeIII]. These emission lines are most likely associated with the Orion HII region (Marconi et al. 1998; Walmsley et al. 2000) and/or the OMC1 molecular hydrogen outflow (e.g. Chernin \& Wright 1996; Kaifu et al. 2000; Nissen et al. 2007). Some contamination from the molecular hydrogen emission can also be seen superposed on the $\mathrm{CO}$ overtone absorption. For the analysis of the reflected absorption spectrum, we have subtracted the nebular spectrum. We estimated the average nebular spectrum by averaging regions of the long slits away from the reflected emission and then subtracted it from each of the absorption spectra so as to remove the $\mathrm{H}_{2}(3,2) \mathrm{S}(2)$ line completely.

The spectrum of region " $\mathrm{d}$ " is very different from the other three because it hardly shows any absorption feature, and the $\mathrm{CO}$ lines are detected in emission. This is consistent with the infrared spectra of the BN object (Scoville et al. 1979), and confirms that the nebular emission close to the BN object is dominated by the reflected light from this source rather than source I within the central KL region, as also inferred from the polarization maps of this region (Minchin et al. 1991). Therefore, we do not consider this spectrum further in the analysis.

The polarization images of regions "a", "b", and "c" suggest that the emission here is dominated by scattered light from the embedded source or sources within the central KL region (Minchin et al. 1991). The three spectra (Fig. 2, top panels) show a common absorption line spectrum. In Fig. 2 we have marked the identification of the most prominent absorption lines. In particular, along with the $\mathrm{CO}(2,0)$ and $\mathrm{CO}(3,1)$ ro-vibrational bands, we clearly detect the CaI triplet at $\sim 2.265 \mu \mathrm{m}$, the $\operatorname{MgI} \lambda 2.281 \mu \mathrm{m}$, and the FeI $\lambda 2.239 \mu \mathrm{m}$ lines.
As mentioned above, our spectra from regions "a", "b", and "c" show a virtually identical absorption line pattern. This result confirms and strengthens the conclusion by Morino et al. (1998) that the absorption spectrum does not originate in the diffuse interstellar medium along the line of sight, but that we are instead observing the absorption spectrum of a single source that is scattered towards us by dust along different lines of sight towards the KL region.

\subsection{Comparison with cool stellar photospheres}

Morino et al. (1998) point out that the reflected absorption spectrum they observed from the region roughly overlapping with our spectrum "a" has an absorption line pattern similar to that of a cool stellar photosphere. Their analysis suggests that the absorption spectrum, especially the atomic lines, is consistent with that of the giant star HD 12014 veiled by an excess featureless continuum emission, which contributes about half of the continuum emission at $\sim 2 \mu \mathrm{m}$. HD 12014 has a K0Ib photosphere, corresponding to an effective temperature of $\sim 4500 \mathrm{~K}$ and low gravity.

Our higher resolution spectra allow a more detailed comparison with stellar photosphere spectral libraries. In Fig. 3 we show the comparison between the spectra in the library of Kleinmann \& Hall (1986) and our average reflected spectrum, obtained by combining the data from regions "a", "b", and "c". Our spectrum was smoothed to the same resolution as the spectra in the library. The photospheric spectra were veiled in such a way to approximately match the depth of the central line of the Ca I triplet in the Orion spectrum. To match the $\mathrm{Ca}$ I absorption the amount of veiling required by the late type spectra is such that the stellar photosphere contributes $\sim 20 \%$ of the computed spectrum, while 

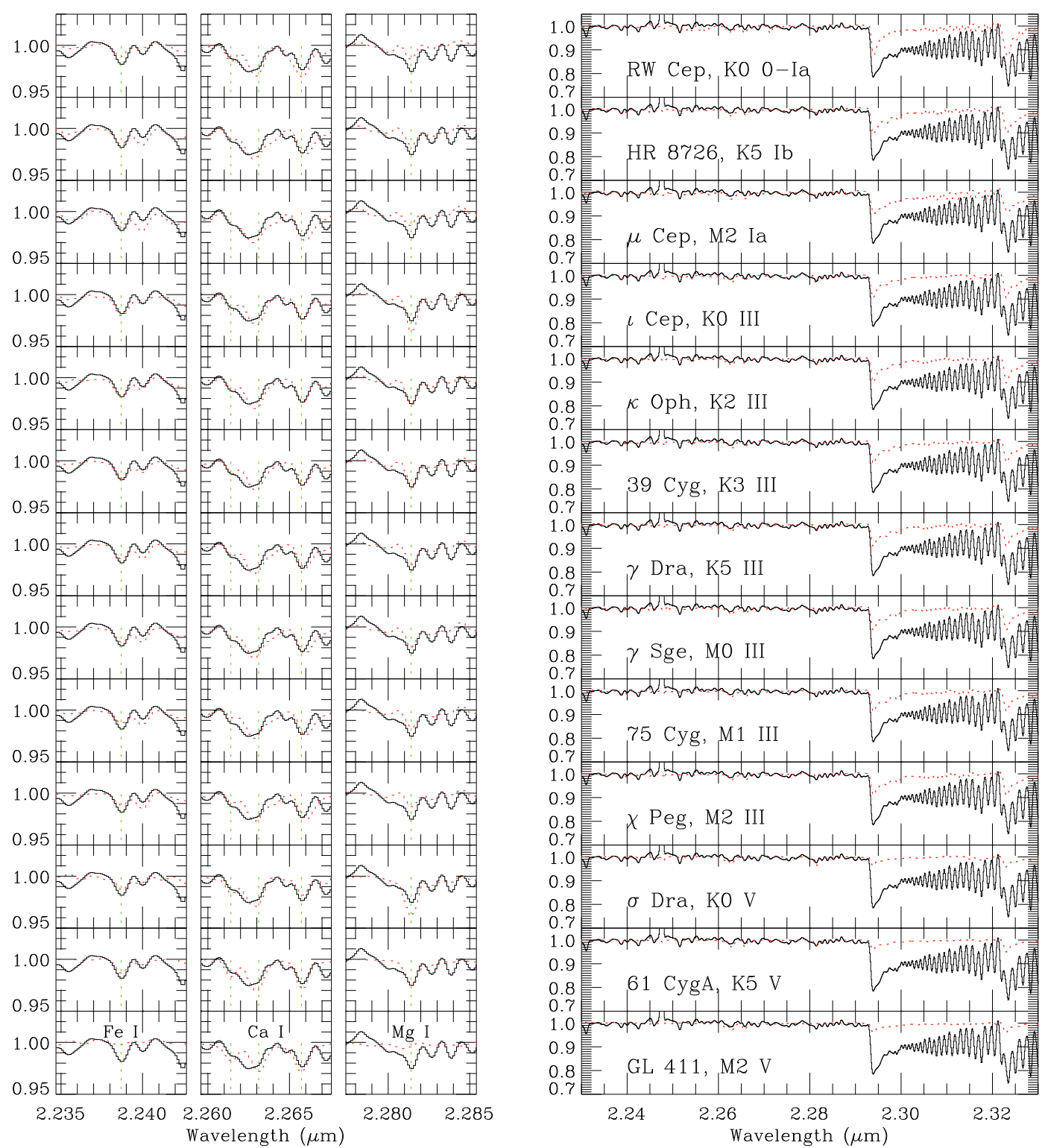

Fig. 3. Comparison between our average reflected spectrum (solid line) and the stellar spectra from Kleinmann \& Hall (1986, red dotted lines). In the left panels we show enlargement around the lines of $\mathrm{Fe} \mathrm{I}, \mathrm{Ca} \mathrm{I}$, and $\mathrm{Mg} \mathrm{I}$ (the wavelength of each feature is marked with a vertical green dotted line), and on the right side we show the full wavelength range of our spectrum, and the name and spectral type of the stars are marked in the right side panels.

for the earlier spectral types the photosphere dominates the spectrum.

The ratio of the atomic absorption lines is best matched with veiled stellar photospheres of spectral types M2-K0, corresponding to effective temperatures in the range $3500-4500 \mathrm{~K}$. Later types have much more pronouced $\mathrm{CaI}$ absorption than the $\mathrm{MgI}$, while the opposite is true for earlier spectral types. In all cases, as noted by Morino et al. (1998), the CO absorption bands are much more pronounced in the Orion spectrum than in the stellar photospheres. In this respect, the lowest gravity, later type spectra in the library are closer to our observed spectrum. This suggests that the spectrum may be consistent with an even lower gravity "photosphere", such as from an accretion disk.

\subsection{Velocity dispersion of the absorption spectra}

To estimate the broadening of the absorption line spectra, we compared the $\mathrm{CO}$ absorption in our ISAAC spectra with the
CO absorption in the high-resolution spectra of Wallace \& Hinkle (1996). The spectra from the library were convolved with a Gaussian of variable width to mimic line broadening (in addition to that intrinsic to the stellar library), shifted in velocity, veiled and then smoothed at the same resolution and resampled onto the same wavelength grid of our ISAAC spectra for proper comparison. These parameters were allowed to vary and a $\chi^{2}$ cube was constructed. In Fig. 4 we show the results of the fit to the spectrum of $\alpha$ Ori, the variation of the $\chi^{2}$ as a function of the $1 \mathrm{D}$ velocity dispersion $(\sigma=F W H M / 1.665)$ of the Gaussian smoothing function for each of the three regions "a", "b", and "c". The results obtained using this star are consistent with those of the other late type, low-gravity stars in the library (such as $\alpha$ Her or RX Boo). The results show that to match our spectra, the photospheric templates need to be smoothed with Gaussian profiles with $\sigma \leq 30 \mathrm{~km} \mathrm{~s}^{-1}$ for region "c", while the spectra of regions "a" and "b" require a much smaller smoothing, $\sigma \leq 15 \mathrm{~km} \mathrm{~s}^{-1}$. The true, total velocity dispersions will be 

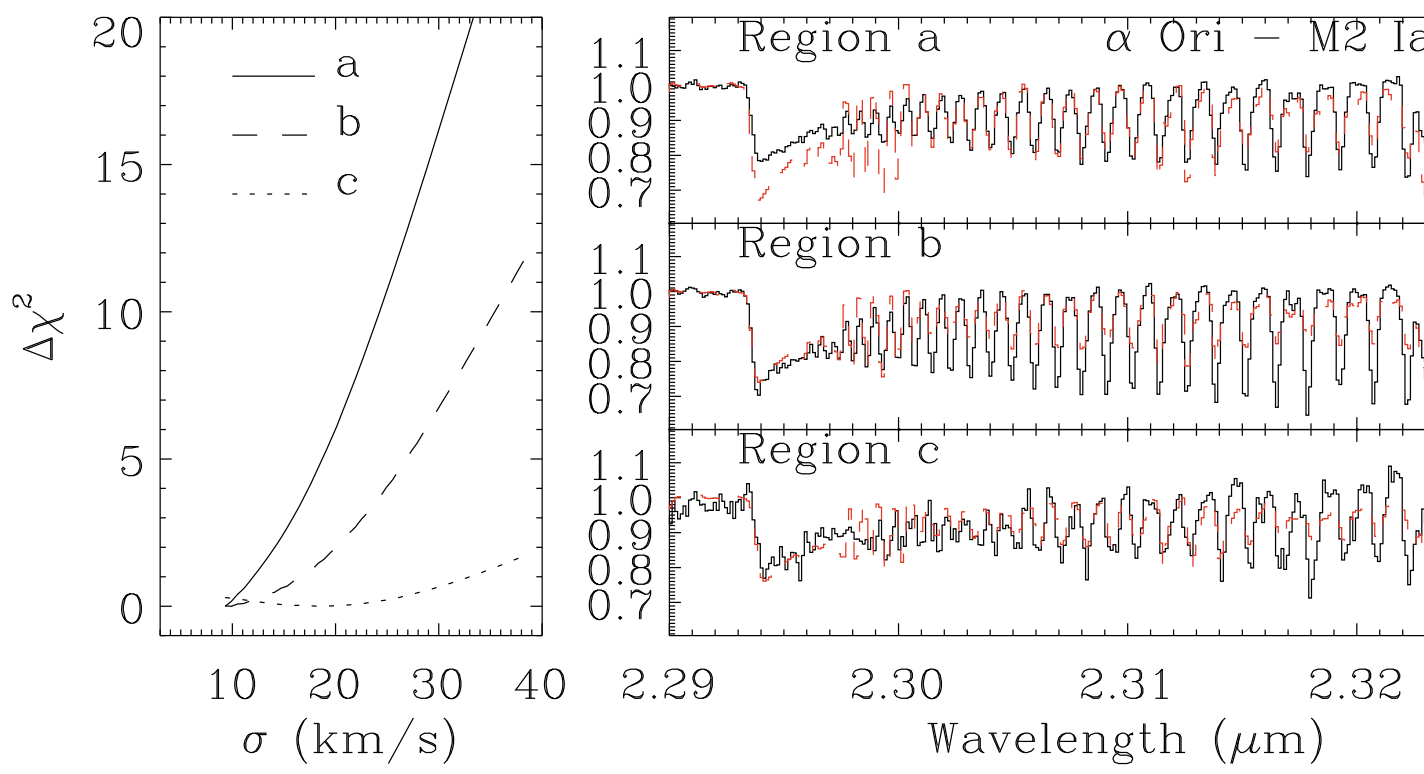

Fig. 4. Results of the $\chi^{2}$ fits to the CO absorption in Orion with the high-resolution infrared spectrum of $\alpha$ Ori (Wallace \& Hinkle 1996). In the left side panel we show the $\Delta \chi^{2}$ curves as a function of the $\Delta V$ used to smooth the library spectrum. On the right we show the comparison between the observed spectrum in each of the three regions (full lines) with the best-fitting photosphere (dashed lines, corresponding to the $\Delta \chi^{2}$ minima in the left-hand side plot).

greater than these limits owing to the contribution of the intrinsic dispersion in the stellar atmospheres used in the spectral library.

Another estimate of the line broadening can be done directly with a simple modeling of the $\mathrm{CO}$ lines. The line parameters were taken from the HITRAN database (Rothman et al. 2009), and line intensities were computed following Helmich (1996) using a simple LTE approximation. As in the procedure with the template spectra, we convolved the model spectra with a Gaussian profile of a given full width at half maximum, and the synthetic spectrum was then scaled to match the observed absorption spectrum, smoothed at the appropriate spectral resolution, and resampled on the same wavelength grid as our ISAAC spectra. This simplified analysis does not allow us to accurately reproduce all the $\mathrm{CO}$ line intensities, but the line broadening required to match the observed spectra is consistent with the results derived using the spectral templates. In particular region "a" requires $\sigma \lesssim 20 \mathrm{~km} \mathrm{~s}^{-1}$, while "b" and "c" are consistent with $\sigma \sim 27 \pm 3 \mathrm{~km} \mathrm{~s}^{-1}$.

We repeated the analysis using a double-peaked line profile as expected from a disk (e.g. Kenyon et al. 1988). Although we do not observe double-peaked lines, our observations would still be consistent with line profiles where the double peaks are separated by $\leq 45 \mathrm{~km} \mathrm{~s}^{-1}$.

The estimates of the linewidths are also consistent with recent observations of mid infrared $\mathrm{CO}$ absorption lines toward a region close to our region "b" (Beuther et al. 2010, their source IRC3). Their spectrum also shows emission components, most likely from outflowing gas, which further complicates the analysis of the line profile.

\section{Discussion}

Our higher resolution spectra $(R \sim 8900$ vs. $\sim 1000)$ allow us to test the different scenarios put forward by Morino et al. (1998), Nakano et al. (2000), and Hosokawa \& Omukai (2009) on the nature of the source producing the absorption spectrum. The latest authors have attempted to explain the observed, cool spectrum as being produced by a very large $\left(r_{*} \gtrsim 200 R_{\odot}\right)$, hence cool
$(T<5500 \mathrm{~K})$, protostar, which requires very high accretion rates averaged over the whole formation time $\left(\dot{m}_{*} \gtrsim 4 \times 10^{-3} M_{\odot} \mathrm{yr}^{-1}\right)$, and the protostar to be at a particular stage in its evolution $\left(m_{*} \simeq 20 M_{\odot}\right)$, undergoing swelling due to the "luminosity wave" (Stahler et al. 1986) immediately before the onset of Kelvin-Helmholz contraction.

For a protostar forming from a near-equilibrium gas core, such high average accretion rates would require very high ambient pressures, equivalent to the self-gravitating weight of a gas clump with $\Sigma \gtrsim 44 \mathrm{~g} \mathrm{~cm}^{-2}$ (McKee \& Tan 2003). However, as described by these authors, the Orion Nebula Cluster has a much lower mean mass surface density of $0.24 \mathrm{~g} \mathrm{~cm}^{-2}$. While moderate enhancements in $\Sigma$, pressure, and thus $\dot{m}_{*}$ are expected in the central regions of the ONC, it appears to be difficult to create the conditions that would allow such high accretion rates to produce the observed NIR scattered light directly from the protostellar surface. The instantaneous accretion rate from a turbulent core is expected to show significant fluctuations about the mean value. Fluctuations may also be induced by tidal interactions with passing stars in the cluster. This scenario is discussed below in the context of source I and the BN object. However, these fluctuations are not expected to significantly change the size of the star, which is set by the accretion rate averaged over the last growth time $\left(m_{*} / \dot{m}_{*}\right)$ of the protostar.

The large protostar scenario makes predictions for the velocity dispersion of the $\mathrm{CO}$ banheads as a function of the rotational velocity of the protostellar surface relative to its breakup velocity. For example a $20 M_{\odot}, 200 R_{\odot}$ protostar rotating at $50 \%$ of the Keplerian velocity at its surface has an equatorial rotation speed of $69 \mathrm{~km} \mathrm{~s}^{-1}$. Viewed at an inclination angle of $45^{\circ}$, this would produces an observed line profile with a velocity dispersion of $\sigma_{\text {rot }} \simeq 50 \mathrm{~km} \mathrm{~s}^{-1}$ (e.g. Gray 1992).

While the large protostar scenario is still marginally consistent with the observed velocity dispersion, we favor the alternative scenario of the NIR spectrum showing CO bandhead absorption being produced in an active accretion disk. There are several arguments in support of this: (1) accretion disks are expected to be present around protostars; (2) the large protostar 
Table 1. Parameters of protostellar models.

\begin{tabular}{ccccccccc}
\hline \hline $\begin{array}{c}m_{*} \\
\left(M_{\odot}\right)\end{array}$ & $\begin{array}{c}r_{*} \\
\left(R_{\odot}\right)\end{array}$ & $\begin{array}{c}L_{*} \\
\left(L_{\odot}\right)\end{array}$ & $\begin{array}{c}T_{*} \\
(\mathrm{~K})\end{array}$ & $\begin{array}{c}\dot{m}_{*} \\
\left(M_{\odot} \mathrm{yr}^{-1}\right)\end{array}$ & $\begin{array}{c}L_{\mathrm{BL}} \\
\left(L_{\odot}\right)\end{array}$ & $\begin{array}{c}L_{\mathrm{tot}} \\
\left(L_{\odot}\right)\end{array}$ & $\begin{array}{c}\sigma \\
\left(\mathrm{km} \mathrm{s}^{-1}\right)\end{array}$ & $\begin{array}{c}\sigma_{\mathrm{irr}} \\
\left(\mathrm{km} \mathrm{s}^{-1}\right)\end{array}$ \\
\hline 8.0 & 12.0 & 5270 & 12200 & $2.40 \times 10^{-4}$ & 2450 & 10200 & 61.0 & 52.7 \\
8.0 & 12.0 & 5270 & 12200 & $2.40 \times 10^{-3}$ & 24500 & 54300 & 40.4 & 36.0 \\
10.0 & 12.5 & 5850 & 14400 & $2.68 \times 10^{-4}$ & 3300 & 12500 & 64.5 & 55.9 \\
10.0 & 12.5 & 5850 & 14400 & $2.68 \times 10^{-3}$ & 33000 & 72000 & 42.7 & 38.0 \\
15.0 & 7.42 & 20100 & 25400 & $3.28 \times 10^{-4}$ & 10300 & 40600 & 69.3 & 56.6 \\
15.0 & 7.42 & 20100 & 25400 & $3.28 \times 10^{-3}$ & 103000 & 226000 & 46.5 & 39.0 \\
20 & 5.81 & 44800 & 35000 & $3.78 \times 10^{-4}$ & 20200 & 85200 & 73.8 & 57.3 \\
\hline
\end{tabular}

scenario requires very high accretion rates and a very special protostellar evolutionary phase; (3) the detection of prominent low excitation $\mathrm{CO}$ absorption near $4.6 \mu \mathrm{m}$ in the reflected spectrum from region "b" (IRC3) by Beuther et al. (2010) suggests there is a range of "photospheric" temperatures. This is expected in an active disk, but difficult to reconcile with a single stellar photospheric temperature - in fact, these low excitation lines are not prominent in cool stellar photospheres (e.g., Sloan et al. 2003); (4) there is evidence that source I is in an active accretion phase, such as the intense outflow activity from the region (e.g. the OMC1 outflow) and the claims that the $\mathrm{cm}$ radio emission and $\mathrm{SiO}$ maser motions around source I indicate the presence of either a compact circumstellar disk (Reid et al. 2007; Matthews et al. 2010) or the ionized base of an outflow cavity (Tan \& McKee 2003).

The accretion disk scenario was also briefly discussed by Nakano et al. (2000). To further explore this possibility, in the next section we compare our spectrum with a more detailed model of a massive protostar-disk system as expected in the turbulent core model for high-mass star formation (McKee \& Tan 2003).

There are claims that the outflow and disk from source I are oriented orthogonally to the orientations assumed in our model, i.e. with the outflow aligned with a NE-SW axis (e.g. Goddi et al. 2009; Reid et al. 2007). Such an orientation is hard to reconcile with the observed orientation of the large-scale outflow from the KL region (Allen \& Burton 1993; Chernin \& Wright 1996) and the morphology of the NIR nebula. We suspect there may still be the possibility of misinterpreting of the outflow and disk orientations from the maser data since the maser emission may not provide a uniform sampling of the gas in these structures. Alternatively, if there has been a strong, recent dynamical interaction of source I with BN (Bally \& Zinnecker 2005; Tan 2004), then the orientation of the disk may have been changed. However, it is still difficult to understand how direct illumination of the NW and SE regions of the KL nebula could be achieved by source I in this scenario, so for these reasons we favor the fiducial model as we have described it.

\subsection{Comparison with the turbulent core protostellar model}

McKee \& Tan (2003) have estimated the range of protostellar models consistent with producing the observed luminosity of the Orion Hot Core, i.e. the Kleinmann-Low Nebula, of $\sim 1-5 \times 10^{4} L_{\odot}$. Here we consider a comparable range of models, with parameters listed in Table 1 . They involve a $60 M_{\odot}$ gas core forming a star with $50 \%$ efficiency and at various stages of collapse, i.e. with $m_{*}$ in the range of 8 to $20 M_{\odot}$. If the core was in pressure equilibrium with a $\Sigma=1 \mathrm{~g} \mathrm{~cm}^{-2}$ surrounding clump, then the accretion rates would be a few $\times 10^{-4} M_{\odot} \mathrm{yr}^{-1}$.

It is possible that the Orion KL protostar has suffered a tidal perturbation in the past $\sim 1000 \mathrm{yr}$ due to the close passage of the runaway BN star (Tan 2004). This is expected to have enhanced the accretion rate through the inner disk and thus also the protostellar mass outflow rate, perhaps causing the apparently "explosive" morpholgy of the outflow (Allen \& Burton 1993). Thus we also consider some models with accretion rates enhanced by a factor of 10, especially for lower mass protostars that will still satisfy the observed total luminosity constraint.

For the $15 M_{\odot}$ protostar accreting at $\dot{m}_{*}=3.28 \times$ $10^{-4} M_{\odot} \mathrm{yr}^{-1}$, the star has a mean spherical radius of $7.42 R_{\odot}$ and internal luminosity of $L_{*}=2.01 \times 10^{4} L_{\odot}$ with a photospheric temperature of $25400 \mathrm{~K}$ (McKee \& Tan 2003). The accretion boundary layer has a luminosity of $L_{\mathrm{BL}}=1.03 \times 10^{4} L_{\odot}$ (with a photospheric temperature of $32000 \mathrm{~K}$ ). The accretion disk is assumed to have the same luminosity as that of the boundary layer. These estimates of accretion luminosity should be regarded as upper limits since they do not include any reduction from the powering of protostellar outflows, which may have mechanical luminosities of about $50 \%$ of the total accretion power.

We assume the protostar has a geometrically thin, optically thick accretion disk. We consider the case of a purely active disk, i.e. with negligible heating of the disk by the protostar, and the case of a protostellar-heated disk, following the treatment of Matzner \& Levin (2005) and Kratter \& Matzner (2006). The reprocessing factor, $f_{\text {irr }}$ is defined as the ratio between the incident flux $F_{\text {irr }}$ normal to the disk surface, and the spherical stellar flux at that radius: $F_{\text {irr }}=f_{\text {irr }} L /\left(4 \pi r^{2}\right)$. An analytic approximation for $f_{\text {irr }}$ is $f_{\text {irr }} \simeq 0.1 \epsilon^{-0.35}$, where $\epsilon$ is the instantaeous star formation efficiency from the core, which we have taken to be $50 \%$ (Matzner \& McKee 2000). In both the non-irradiated and irradiated cases, emission from the accretion disk dominates the emergent NIR flux, as shown in Fig. 5.

The radial profiles of disk photospheric temperature and Keplerian velocity are shown in Fig. 6. We evaluated the contribution to the total $\mathrm{CO}$ line profile from each emitting part of the disk, weighting by the disk area, flux at $2.3 \mu \mathrm{m}$, and a temperature-dependent "CO bandhead weighting factor", $f_{\mathrm{CO}}(T)$, which is derived from the observed equivalent widths of giant and supergiant standard stars (see Fig. 7). This factor is uncertain at $T<2000 \mathrm{~K}$, although we have checked various functional forms that decline with temperature for this temperature range and found that our results are not particularly sensitive to such variations. We assume a typical inclination angle of $45^{\circ}$ of dust particles to the rotation axis of the accretion disk, since the PA of the axis of the bipolar flow (and the observed near IR reflection nebulosity) is approximately from the NW to the SE (e.g. Chernin \& Wright 1996). The application of this outflow geometry to source I is consistent with the interpretation of source I's radio emission as the ionized base of an outflow cavity (Tan \& McKee 2003), but not with the interpretation that the disk axis is oriented from the NW to SE (Reid et al. 2007; Matthews et al. 2010). 


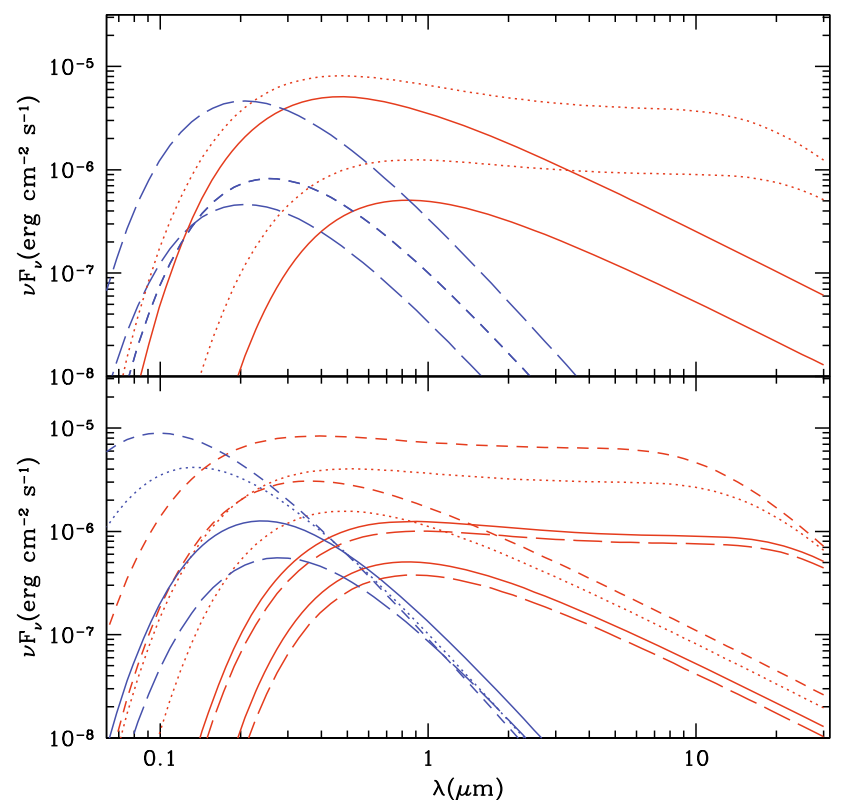

Fig. 5. Spectra of protostellar models (see text). Top panel: for the fiducial accretion rate model with $m_{*}=10 M_{\odot}$, the fluxes from the star and boundary layer are shown by the dashed and lower long-dashed lines, respectively. The lower solid line shows the flux from the accretion disk (integrating out to $5000 r_{*}$ ) assuming negligible protostellar heating, while the lower dotted line shows the case accounting for protostellar heating. The upper long-dashed, solid, and dotted lines show the equivalent quantities for the model with the accretion rate enhanced by a factor of 10 . Bottom panel: the solid lines show for the fiducial $m_{*}=10 M_{\odot}$ model the flux from the star+boundary layer, nonirradiated and irradiated accretion disks. The long-dashed, dotted, and dashed lines show the equivalent fluxes from the fiducial accretion rate models with $m_{*}=8,15$, and $20 M_{\odot}$, respectively. In all cases, the flux at $2.3 \mu \mathrm{m}$ is dominated by disk emission.

The $1 \mathrm{D}$ velocity dispersions, $\sigma$, of the resulting line profiles are listed in Table 1. The irradiated disks have lower values caused by the disk being warmer at a given radius and the $\mathrm{CO}$ emitting region being located at larger radii. If an inclination angle of $30^{\circ}$ is adopted, for example, for the $15 M_{\odot}$ model with $\dot{m}_{*}=3.28 \times 10^{-4} M_{\odot} \mathrm{yr}^{-1}$, then the velocity dispsersion drops to $\sigma=49.1 \mathrm{~km} \mathrm{~s}^{-1}$ and $\sigma_{\text {irr }}=40.1 \mathrm{~km} \mathrm{~s}^{-1}$.

The models with enhanced accretion rates cause the disk to be warmer at a given radius, thus pushing the $\mathrm{CO}$ bandhead region of the disk to larger radii and lower Keplerian velocities. This produces lower values of $\sigma$.

We conclude that the fiducial turbulent core protostellar models (i.e. those without enhanced accretion rates) for the Orion KL protostar predict $\mathrm{CO}$ bandhead 1D observed velocity dispersions that are in the range 53 to $75 \mathrm{~km} \mathrm{~s}^{-1}$. The velocity dispersion is not very sensitive to $m_{*}$ because the temperature range that contributes to $\mathrm{CO}$ bandhead absorption, i.e. $T \sim 2000-4000 \mathrm{~K}$ occurs at a fairly constant range of Keplerian velocities in the accretion disk, nearly independent of $m_{*}$. Disk irradiation raises the temperature of the disk at any given radius, thus shifting the zone that contributes to $\mathrm{CO}$ bandhead equivalent width to larger radii and thus yielding narrower line profiles. The velocity dispersion of the line profile is somewhat sensitive to the adopted inclination angle between the scattering surfaces in the outflow and the disk rotation axis. The fiducial protostellar models predict 1D CO bandhead velocity dispersions that are about a factor of two to three greater than observed. A better match is achieved if there is a relatively stronger contribution to $\mathrm{CO}$ bandhead EW from lower velocity dispersion gas, which

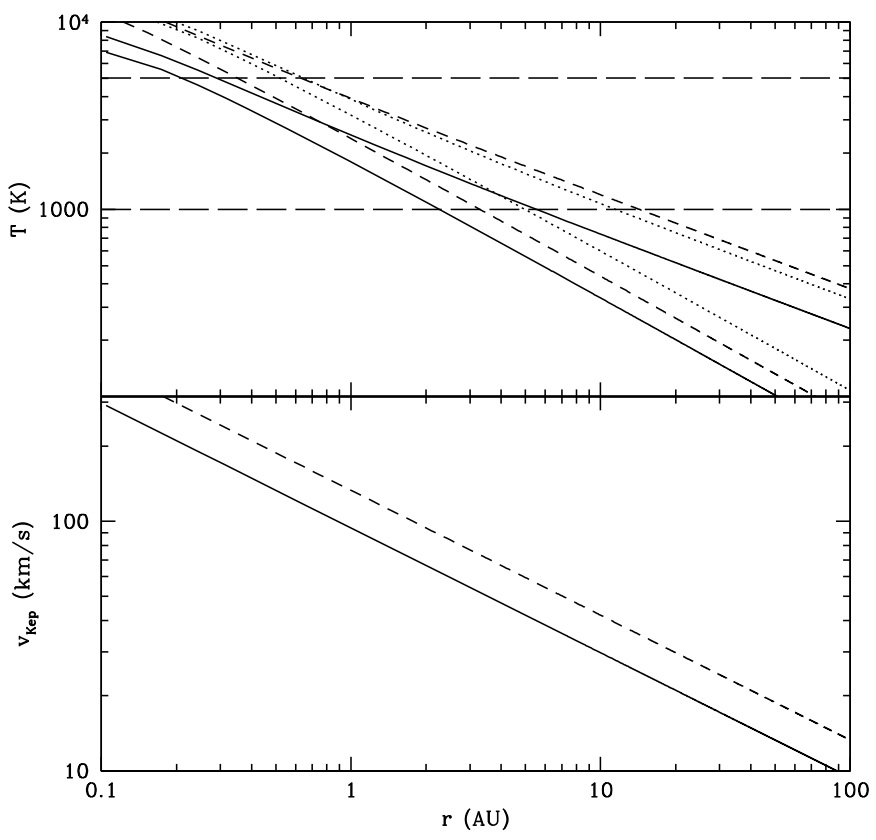

Fig. 6. Radial profiles of protostellar accretion disk models. Top panel: disk photospheric temperature for non-irradiated disk (lower solid line) and irradiated disk (upper solid line) for $m_{*}=10 M_{\odot}$ with fiducial accretion rate. The equivalent models for $m_{*}=10 M_{\odot}$ with accretion rate enhanced by a factor of 10 are shown by the dotted lines. The equivalent models for $m_{*}=20 M_{\odot}$ with fiducial accretion rate are shown by the dashed lines. The long-dashed horizontal lines show the approximate range of temperatures where we consider there are contributions to the CO bandhead equivalent width. Bottom panel: Keplerian velocities for the $m_{*}=10,20 M_{\odot}$ models, shown by the solid and dashed lines, respectively.

can be reproduced in models with accretion rates enhanced by a factor of 10 . Higher velocity resolution observations of the scattered light from Orion KL are needed to better establish the significance of the observed velocity dispersion in order to further test theoretical models, including the search for double-peaked line profiles.

\section{Summary and conclusions}

In this paper we have presented medium-resolution $(R \sim 8900)$, near-infrared spectra of the reflection nebulosity in Orion-KL. Our main results are as follows.

- The absorption line spectra in various regions of the reflection nebula in Orion-KL are consistent with each other, except for a small difference in the line broadening. This suggests a common origin for the absorption line close to the region emitting the continuum emission. We thus confirm the earlier suggestion by Morino et al. (1998) that the absorption spectrum is generated close to the position of source I, either from the protostellar photosphere or the surrounding accretion disk. This also suggests that the outflow cavity from the main illuminating source in the KL nebula, i.e. source I, is aligned from the SE to the NW, which is difficult to explain in models that invoke a disk plane along this axis (Reid et al. 2007; Matthews et al. 2010).

- The observed absorption spectra are broadly consistent with low-gravity, late-type, heavily veiled stellar photospheres with spectral types in the range M2-K0 and luminosity class I-III, corresponding to effective temperatures in the range $3500-4500 \mathrm{~K}$. Nevertheless, it was not possible to find a good match for all the observed absorption features with a 


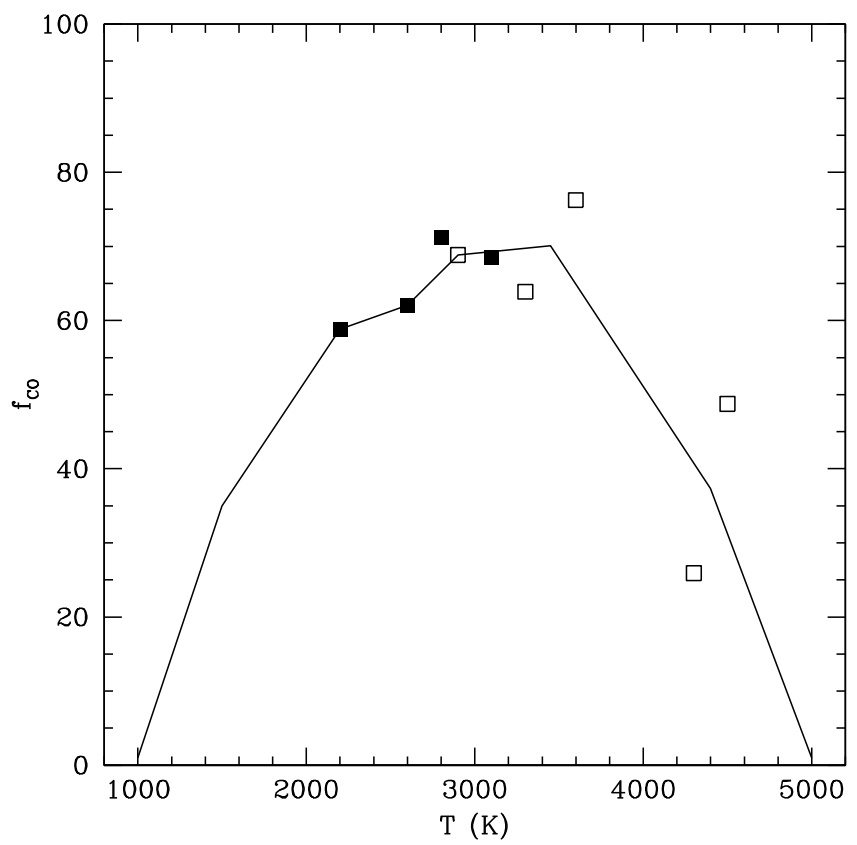

Fig. 7. Weighting factor of $\mathrm{CO}(2-0)$ bandhead equivalent width over the wavelength range $2.292-2.322 \mu \mathrm{m}$ derived from giant/supergiant standard spectra (open squares) and Mira spectra scaled by a factor of 0.7 (solid squares).

single photospheric template. In particular, the observed $\mathrm{CO}$ absorption in the Orion spectra are much deeper than in the stellar photospheres that match the atomic lines. Although it is possible that an even lower gravity photosphere than those used in the comparison may alleviate or solve this inconsistency, it is likely that this discrepancy could be solved if the Orion spectrum is produced by the combination of a wide range of photospheric temperatures, as in a very active accretion disk.

- The observed line profiles are consistent with being unresolved at our spectral resolution in regions "a" and "b" and marginally resolved in region "c". These results imply $\sigma \sim 30 \mathrm{~km} \mathrm{~s}^{-1}$ for region "c" and about a factor of two less for regions "a" and "b". We thus exclude that the spectra could be originated from the photosphere of a massive $\left(\sim 20 M_{\odot}\right)$, large $\left(\sim 200 R_{\odot}\right)$ protostar rotating close to the breakup speed. The linewidths are consistent with the predictions of a model of an active accretion disk around a massive protostar $\left(M \sim 10 M_{\odot}\right)$. Models with accretion rates enhanced above the fiducial values are favored in order to reproduce the relatively narrow line widths.

We conclude that the most likely source of the near infrared absorption spectrum is the accretion disk surrounding the massive protostar source I, with the system in a state of relatively high accretion rate.
Acknowledgements. We thank the ESO support astronomers and La Silla Paranal Observatory staff for their support in the preparation and execution of the service mode observations for this program. This work was partially supported through ASI grants to the INAF-Osservatorio Astrofisico di Arcetri. J.C.T. acknowledges support from NSF CAREER grant AST-0645412 and the ESO visitor program.

\section{References}

Allen, D. A., \& Burton, M. G. 1993, Nature, 363, 54 Bally, J., \& Zinnecker, H. 2005, AJ, 129, 2281

Becklin, E. E., \& Neugebauer, G. 1967, ApJ, 147, 799

Beuther, H., Churchwell, E. B., McKee, C. F., \& Tan, J. C. 2007, Protostars and Planets V, 165

Beuther, H., Linz, H., Bik., A., Goto, M., \& Henning, T. 2010, A\&A, 512, A29 Chernin, L. M., \& Wright, M. C. H. 1996, ApJ, 467, 676

Gezari, D. Y., Backman, D. E., \& Werner, M. W. 1998, ApJ, 509, 283

Goddi, C., Greenhill, L. J., Chandler, C. J., et al. 2009, ApJ, 698, 1165

Gómez, L., Rodríguez, L. F., Loinard, L., et al. 2008, ApJ, 685, 333

Gray, D. F. 1992, The observation and analysis of stellar photospheres, ed. D. F. Gray

Helmich, F. P. 1996, Ph.D. Thesis, Leiden University

Hosokawa, T., \& Omukai, K. 2009, ApJ, 691, 823

Kaifu, N., Usuda, T., Hayashi, S. S., et al. 2000, PASJ, 52, 1

Kenyon, S. J., Hartmann, L., \& Hewett, R. 1988, ApJ, 325, 231

Kleinmann, D. E., \& Low, F. J. 1967, ApJ, 149, L1

Kleinmann, S. G., \& Hall, D. N. B. 1986, ApJS, 62, 501

Kratter, K. M., \& Matzner, C. D. 2006, MNRAS, 373, 1563

Marconi, A., Testi, L., Natta, A., \& Walmsley, C. M. 1998, A\&A, 330, 696

Matthews, L. D., Greenhill, L. J., Goddi, C., et al. 2010, ApJ, 708, 80

Matzner, C. D., \& Levin, Y. 2005, ApJ, 628, 817

Matzner, C. D., \& McKee, C. F. 2000, ApJ, 545, 364

McKee, C. F., \& Tan, J. C. 2003, ApJ, 585, 850

Menten, K. M., \& Reid, M. J. 1995, ApJ, 445, L157

Minchin, N. R., Hough, J. H., McCall, A., et al. 1991, MNRAS, 248, 715

Morino, J.-I., Yamashita, T., Hasegawa, T., \& Nakano, T. 1998, Nature, 393, 340

Nakano, T., Hasegawa, T., Morino, J.-I., \& Yamashita, T. 2000, ApJ, 534, 976

Nissen, H. D., Gustafsson, M., Lemaire, J. L., et al. 2007, A\&A, 466, 949

Plambeck, R. L., Wright, M. C. H., \& Carlstrom, J. E. 1990, ApJ, 348, L65

Plambeck, R. L., Wright, M. C. H., Mundy, L. G., \& Looney, L. W. 1995, ApJ, 455, L189

Plambeck, R. L., Wright, M. C. H., Friedel, D. N., et al. 2009, ApJ, 704, L25

Reid, M. J., Menten, K. M., Greenhill, L. J., \& Chandler, C. J. 2007, ApJ, 664, 950

Rieke, G. H., Low, F. J., \& Kleinmann, D. E. 1973, ApJ, 186, L7

Rothman, L. S., Gordon, I. E., Barbe, A., et al. 2009, J. Quant. Spectrosc. Radiat. Transfer, 110, 533

Scoville, N. Z., Hall, D. N. B., Ridgway, S. T., \& Kleinmann, S. G. 1979, ApJ, 232, L121

Shu, F. H., Adams, F. C., \& Lizano, S. 1987, ARA\&A, 25, 23

Sloan, G. C., Kraemer, K. E., Price, S. D., \& Shipman, R. F. 2003, ApJS, 147, 379

Stahler, S. W., Palla, F., \& Salpeter, E. E. 1986, ApJ, 302, 590

Tan, J. C. 2004, ApJ, 607, L47

Tan, J. C. 2008, ApJL, submitted [arXiv: 0807.3771]

Tan, J. C., \& McKee, C. F. 2003, [arXiv:astro-ph/0309139v1]

Wallace, L., \& Hinkle, K. 1996, ApJS, 107, 312

Walmsley, C. M., Natta, A., Oliva, E., \& Testi, L. 2000, A\&A, 364, 301

Werner, M., Capps, R., \& Dinerstein, H. 1983, ApJ, 265, L13

Yorke, H. W., \& Bodenheimer, P. 2008, in Massive Star Formation: Observations

Confront Theory, ed. H. Beuther, H. Linz, \& T. Henning, ASP Conf. Ser., 387, 189

Zinnecker, H., \& Yorke, H. W. 2007, ARA\&A, 45, 481 